\title{
Adult-onset immunodeficiency - why is it important in rheumatology?
}

\author{
Hans-Hartmut Peter*
}

When the editors of Arthritis Research \& Therapy asked me to coordinate a review series on adult immunodeficiency, I voluntarily agreed and suggested 10 topics. The editors selected six with the greatest importance for rheumatologists. The authors of the six articles all come from research groups with outstanding track-records in their respective fields.

Why present adult-onset immunodeficiency to a readership of rheumatologists? The answer is simple. First, immunodeficiency is a booming field of translational immunology that should not be ignored by the rheumatologist. Second, there are increasing numbers of patients with symptoms of immunodeficiency being seen in rheumatology clinics. Rheumatologists are often confronted with patients suffering from either systemic autoimmune diseases plus drug-induced increased susceptibility to infections or with alarming symptoms or with unusual laboratory values all indicative of a primary dysfunction of the immune system. Alarming symptoms include recurrent bouts of fever, frequent and prolonged infections, unusual pathogens, hepatosplenomegaly, lymphadenopathy, recurrent mucocutaneous candidiasis, spontaneous bone fractures, lung abscesses and noncaseating granulomas. Typical laboratory findings are leukopenia, lymphopenia, low CD4 counts, myelodysplasia, autoimmune hemolytic anemia and thrombocytopenia as well as low concentrations of serum immunoglobulins or high IgE or IgM serum levels.

Who is best suited to take care of these patients in our current medical systems - the general physician, the hematologist, the infectious disease expert, the clinical immunologist or the rheumatologist? My honest view is that modern rheumatology, with its intricate links to clinical immunology, is the medical discipline best prepared to follow these patients and organize a close consultation platform with other disciplines, notably hematology, infectious disease and pathology. Along

\footnotetext{
*Correspondence: hans-hartmut.peter@uniklinik-freiburg.de Center of Chronic Immunodeficiency, Engesserstraße 4, D-79108 Freiburg, Germany
}

these lines, rheumatologists, clinical immunologists, pediatricians, infectious disease experts and pathologists have recently founded a unique Center of Chronic Immunodeficiency at the Freiburg University Medical Center in Germany. The Center covers a hitherto unmet need for expert diagnosis and treatment of immunodysregulatory and immunodeficiency syndromes both in children and adults. Up to 10 new patients are seen every week. Many of these patients suffer from an established or a novel genetic defect. About $40 \%$ of the patients with proven monogenic immunodeficiency syndromes also present with symptoms of systemic or localized autoimmunity. Rheumatologists should therefore become acquainted with the notion that systemic collagen-vascular disease and inflammatory joint diseases are likely to belong to a spectrum of immunodysregulatory syndromes that comprise, in addition to autoimmunity and immunodeficiency, nonmalignant lymphoproliferation and granulomatous disease.

The clinical phenotype in these patients can be dominated by either autoimmunity or immunodeficiency, depending on the mixture of polymorphisms or mutations and deletions in genes involved in the innate and adaptive immune response. Environmental, metabolic and epigenetic factors can add to the complexity of the underlying pathophysiology. A prominent example of this situation relates to ICOS deficiency (inducible T-cell costimulator; CD278): a deletion mutation described in Europe exhibits the classical phenotype of common variable immunodeficiency [1], whereas a different mutation in the same gene reported in Japan associates more with autoimmunity symptoms [2]. Similar findings have been observed for mutations in the gene coding for the Wiskott-Aldrich syndrome protein: depending on the site of the mutation, either X-linked thrombocytopenia or the full-blown Wiskott-Aldrich syndrome phenotype can occur [3]. The list of such genotype-phenotype relationships oscillating between immunodeficiency and autoimmunity is long.

In the present review series on adult immunodeficiency, six topics of particular interest to pediatric and adult rheumatology have been selected. 
A problem encountered quite often in rheumatology is a low CD4 count with or without opportunistic infections. The differential diagnosis is demanding: HIV infection but also systemic lupus erythematosus, Sjögren's syndrome, primary immunodeficiencies, hemophagocytic syndromes, idiopathic CD4 lymphopenia and druginduced effects may all hide behind this scenario. Zonios and colleagues have critically reviewed this challenging topic [4].

Remarkable scientific progress has been made on hyperIgM syndromes and hypogammaglobulinemia/common variable immunodeficiency. Durandy and Kracker [5] and Salzer and colleagues [6] have expertly reviewed each field respectively and pointed out their relevance for rheumatology.

Similarly, macrophage activation syndromes that lead to life-threatening hemophagocytic lymphohistiocytosis are of great interest for rheumatologists. These conditions can afflict children and adults as familial lymphohistiocytic hemophagocytosis due to typical mutations in genes controlling vesicle transport in cytotoxic lymphocytes, or they can occur idiopathically in the context of childhood or adult-onset Still's disease, persistent viral infections (Epstein-Barr virus, cytomegalovirus) or drug hypersensitivity. These patients require a fast and competent diagnosis and an early identification of individuals who need to be directed towards allogeneic stem cell transplantation - the only curative procedure available. Ehl's group has reviewed the new developments in this fascinating field [7].

Finally, rheumatologists will profit from an increased awareness of two important areas of recent discoveries: first, a series of gene defects leading to deregulated IL-17 responses in chronic mucocutaneous candidiasis reviewed by Gaffen's group [8]; and, second, the crucial importance of mutations in the signal transduction molecules STAT3 and DOCK8 in hyper-IgE syndrome reviewed by Yong and colleagues [9].

The editors' decision to offer this review series to a rheumatology readership is both daring and visionary: daring because it will challenge rheumatologists to engage in new clinical areas, and visionary because it aims at directing rheumatology deeper into clinical immunology, immunodysregulation and novel strategies of targeted therapies. The authors of this issue are convinced that the chosen direction is an important asset for modern rheumatology.

This article is part of the series on Adult immunodeficiency, edited by Hans-Hartmut Peter. Other articles in this series can be found at http://arthritis-research.com/series/immunodeficiency

\section{Abbreviations}

DOCK, dedicator of cytokinesis; ICOS, inducible T-cell costimulator (CD278); IL, interleukin; STAT, signal transducer and activator of transcription.

\section{Competing interests}

The author declares that he has no competing interests.

Published: 30 January 2013

\section{References}

1. Grimbacher B, Hutloff A, Schlesier M, Glocker E, Warnatz K, Drager R, Eibel H, Fischer B, Schaffer AA, Mages HW, Kroczek RA, Peter HH: Homozygous loss of ICOS is associated with adult-onset common variable immunodeficiency. Nat Immuno/ 2003, 4:261-268.

2. Takahashi N, Matsumoto K, Saito H, Nanki T, Miyasaka N, Kobata T, Azuma M, Lee SK, Mizutani S, Morio T: Impaired CD4 and CD8 effector function and decreased memory T cell populations in ICOS-deficient patients. J Immunol 2009, 182:5515-5527.

3. Albert MH, Bittner TC, Nonoyama S, Notarangelo LD, Burns S, Imai K, Espanol T, Fasth A, Pellier I, Strauss G, Morio T, Gathmann B, Noordzij JG, Fillat C, Hoenig M, Nathrath M, Meindl A, Pagel P, Wintergerst U, Fischer A, Thrasher AJ, Belohradsky BH, Ochs HD: X-linked thrombocytopenia (XLT) due to WAS mutations: clinical characteristics, long-term outcome, and treatment options. Blood 2010, 115:3231-3238.

4. Zonios D, Sheikh V, Sereti J: A case of missing, wandering or ineffective T cells. Arthritis Res Ther 2012, 14:222.

5. Durandy A, Kracker S: Immunoglobulin class switch recombination deficiencies. Arthritis Res Ther 2012, 14:218.

6. Salzer U, Warnatz K, Peter HH:Common variable immunodeficiency (CVID) an update. Arthritis Res Ther 2012, 14:223. doi: 10.1186/ar4032

7. Bode SFN, Lehmberg K, Maul-Pavesic A, Vraetz T, Janka G, zur Stadt U, EhI S: Recent advances in diagnosis and treatment of hemophagocytic lymphohistiocytosis. Arthritis Res Ther 2012, 14:213.

8. Huppler A, Bishu S, Gaffen SH: Mucocutaneous candidiasis. The IL17 pathway and its implications to targeted therapy. Arthritis Res Ther 2012, $14: 217$.

9. Yong PFK, Freeman AF, Engelhardt K, Holland S, Puck J, Grimbacher B: An update on hyper-IgE syndrome. Arthritis Res Ther 2012, 14:228.

doi:10.1186/ar4121

Cite this article as: Peter HH: Adult-onset immunodeficiency - why is it

important in rheumatology? Arthritis Research \& Therapy 2013, 15:105. 